\title{
THE DENSITY CHARACTER OF UNIONS
}

\author{
W. W. COMFORT ${ }^{1}$ AND TEKLEHAIMANOT RETTA
}

\begin{abstract}
We consider only completely regular, Hausdorff spaces. Responding to a question of R. Levy and R. H. McDowell [Proc. Amer. Math. Soc. 49 (1975), 426-430] we show that for $\omega<\gamma<2^{2 \omega}$ there is a separable space equal to the (appropriately topologized) disjoint union of $\gamma$ copies of the "Stone-Cech remainder" $\beta N \backslash N$. More generally, denoting density character by $d$ and weight by $w$, we prove this

THEOREM. The following statements about infinite cardinal numbers $\gamma$ and $\alpha$ are equivalent: (a) $2^{\alpha}<2^{\gamma}$ and $\gamma<2^{2^{\alpha}}$; (b) For every family $\left\{X_{\xi}: \xi<\gamma\right\}$ of spaces, with $w\left(X_{\xi}\right)<2^{\alpha}$ for all $\xi<\gamma$, the set-theoretic disjoint union $X=\bigcup_{\xi<\gamma} X_{\xi}$ admits a topology such that $d(X)<\alpha$ and each $X_{\xi}$ is a topological subspace of $X$.

The following observation (a special case of Theorem 3.1) suggests that it may be difficult to achieve a stronger result: If $\alpha>\omega$ and $X_{0}$ and $X_{1}$ denote copies of the discrete space of cardinality $\alpha^{+}$, then the disjoint union $X=X_{0} \cup X_{1}$ admits a topology (making each $X_{i}$ a topological subspace) such that $d(X)<\alpha$.
\end{abstract}

1. Notation and references to the literature. By a "space" we mean a completely regular, Hausdorff space. The symbols $d$ and $w$ were defined in the abstract. For $\alpha \geqslant \omega$ we set

$$
\log \alpha=\min \left\{\gamma: 2^{\gamma} \geqslant \alpha\right\} .
$$

When $\alpha \geqslant \omega$ we denote also by the symbol $\alpha$ the discrete space of cardinality $\alpha$, and by $\boldsymbol{\beta}(\alpha)$ its Stone-Čech compactification. As usual we identify $\boldsymbol{\beta}(\alpha)$ with the set of ultrafilters on $\alpha$, topologized so that

$$
\{\{p \in \boldsymbol{\beta}(\alpha): A \in p\}: A \subset \alpha\}
$$

is a base for the closed sets; evidently $w(\beta(\alpha)) \leqslant 2^{\alpha}$, so that $w(X) \leqslant 2^{\alpha}$ for all $X \subset \boldsymbol{\beta}(\alpha)$. We set

$$
U(\alpha)=\{p \in \beta(\alpha):|A|=\alpha \text { for all } A \in p\},
$$

and we recall (see for example Corollary 7.15 of [1]) that there are $p \in U(\alpha)$ with no basis of cardinality $<2^{\alpha}$. Thus we have:

1.1. If $\alpha \geqslant \omega$, then $w(U(\alpha))=2^{\alpha}$.

Received by the editors April 12, 1976 and, in revised form, October 29, 1976.

AMS (MOS) subject classifications (1970). Primary 54A25; Secondary 54A10.

Key words and phrases. Density character, weight, Tychonoff space.

'This author gratefully acknowledges support received from the National Science Foundation under grant NSF-MPS73-08937A02. 
It is well known and easy to prove that for every space $X$ the family of regular-open subsets of $X$ (i.e., the family of subsets $U$ of $X$ such that $U=$ int cl $U$ ) is a base for $X$. Further, if $D$ is dense in $X$ and $U$ and $V$ are different regular-open subsets of $X$, then $U \cap D \neq V \cap D$. This proves 1.2 below. Statements 1.3, 1.4 and 1.5 are equally familiar. For proofs, see for example Corollaries 2.11, 3.18, and 12.20 (together with Lemma 7.12 (b)) of [1].

1.2. If $X$ is a space then $w(X) \leqslant 2^{d(X)}$.

We denote the real line in its usual topology by the symbol $\mathbf{R}$.

1.3. If $X$ is a space such that $w(X) \leqslant \alpha$, then $X$ is (homeomorphic with) a subspace of $\mathbf{R}^{\alpha}$; thus $|X| \leqslant 2^{w(X)}$.

1.4. If $\alpha \geqslant \omega$ then $d\left(\mathbf{R}^{2^{\alpha}}\right) \leqslant \alpha$.

1.5. If $\alpha \geqslant \omega$ and $V$ is a nonempty, open subset of $U(\alpha)$, then there is a family $\mathcal{U}$ of pairwise disjoint, nonempty open subsets of $V$ such that $|थ|=\alpha^{+} ;$hence $d(V)>\alpha$.

2. Topologizing a disjoint union. If $X_{\xi}$ is a subspace of a space $X$ such that $d(X) \leqslant \alpha$, then from 1.2 above we have $w\left(X_{\xi}\right) \leqslant w(X) \leqslant 2^{\alpha}$. This explains the presence of the hypothesis " $w\left(X_{\xi}\right) \leqslant 2^{\alpha}$ " in the following result.

2.1. TheOREM. Let $\alpha$ and $\gamma$ be cardinals, with $\alpha>\omega$. The following statements are equivalent.

(a) $\log 2^{\alpha} \leqslant \gamma \leqslant 2^{2^{\alpha}}$

(b) for every family $\left\{X_{\xi}: \xi<\gamma\right\}$ of (pairwise disjoint) nonempty spaces, with $w\left(X_{\xi}\right) \leqslant 2^{\alpha}$ for all $\xi<\gamma$, the set-theoretic disjoint union $X=\cup_{\xi<\gamma} X_{\xi}$ admits a topology such that $d(X) \leqslant \alpha$ and each $X_{\xi}$ is a topological subspace of $X$.

Proof. (a) $\Rightarrow$ (b). Let $w\left(X_{\xi}\right) \leqslant 2^{\alpha}$ for all $\xi<\gamma$, define $\delta=\log 2^{\alpha}$, using 1.4 above let $D=\{p(\xi): \xi<\delta\}$ be a faithfully indexed dense subset of $\mathbf{R}^{2^{\alpha}}$, and choose $p(\delta) \in \mathbf{R}^{2^{\alpha}} \backslash D$. For $S \subset 2^{\alpha}$ we denote by $\pi_{S}$ the projection from $\mathbf{R}^{2^{\alpha}}$ onto $\mathbf{R}^{S}$, and we choose $A \subset 2^{\alpha}$ such that $|A|=\delta$ and $\pi_{A} \mid D \cup\{p(\delta)\}$ is a one-to-one function. For $\xi \leqslant \delta$ we define

$$
G_{\xi}=\pi_{A}^{-1}\left(\pi_{A}(p(\xi))\right)=\left\{x \in \mathbf{R}^{2^{\alpha}}: x_{\eta}=p(\xi)_{\eta} \text { for all } \eta \in A\right\},
$$

and we note (since $\delta \leqslant \alpha<2^{\alpha}$ ) that $G_{\xi}$ is homeomorphic to $\mathbf{R}^{2^{\alpha}}$. It follows from 1.3 above that for $\xi<\delta$ the space $X_{\xi}$ is (homeomorphic with) a subspace of $G_{\xi}$; we assume without loss of generality, using the fact that $G_{\xi}$ is a homogeneous space, that $p(\xi) \in X_{\xi}$ for all $\xi<\delta$.

If $\gamma=\delta$ then since $D$ is dense in $\mathbf{R}^{2^{\alpha}}$ and

$$
D \subset X=\bigcup_{\xi<\gamma} X_{\xi} \subset \bigcup_{\xi<\gamma} G_{\xi} \subset \mathbf{R}^{2^{\alpha}}
$$

we have $d(X) \leqslant \alpha$ and the proof is complete. If $\delta<\gamma \leqslant 2^{2^{\alpha}}$ then we note that since $G_{\delta}$ is homeomorphic with $\mathbf{R}^{2^{\alpha}}$, hence with $\mathbf{R}^{2^{\alpha}} \times \mathbf{R}^{2^{\alpha}}$, the space $G_{\delta}$ contains $\gamma$ disjoint copies (indeed, $2^{2^{\alpha}}$ disjoint copies) of $\mathbf{R}^{2^{\alpha}}$. Thus the spaces $X_{\xi}$ (with $\delta \leqslant \xi<\gamma$ ) are homeomorphic with pairwise disjoint subspaces of $G_{\delta}$ 
and again, giving $X=\cup_{\xi<\delta} X_{\xi}$ the topology inherited from $\mathbf{R}^{2^{a}}$, we have $d(X) \leqslant \alpha$ because $D \subset X \subset \mathbf{R}^{2^{\alpha}}$ and $D$ is dense in $\mathbf{R}^{2^{\alpha}}$.

(b) $\Rightarrow$ (a). From 1.2 and 1.3 we have $|X| \leqslant 2^{2^{d(X)}}$, so that necessarily $\gamma \leqslant 2^{2^{a}}$.

Let $\gamma<\log 2^{\alpha}$, let $X_{0}=U(\alpha)$ and for $0<\xi<\gamma$ let $X_{\xi}$ be the singleton space $\{\xi\}$, suppose that the set-theoretic disjoint union $X=\cup_{\xi<\gamma} X_{\xi}$ is topologized as in (b), and let $D$ be a dense subset of $X$ such that $|D| \leqslant \alpha$. If $D \backslash X_{0}$ is dense in $X$ then we have $d(X) \leqslant \gamma$ and hence (from 1.1 and 1.2),

$$
2^{\alpha}=w\left(X_{0}\right) \leqslant w(X) \leqslant 2^{d(X)} \leqslant 2^{\gamma}<2^{\alpha},
$$

a contradiction. Thus there is a nonempty, open subset $V$ of $X_{0}$ such that $V \subset \mathrm{cl}_{X_{0}}\left(D \cap X_{0}\right)$, so that $V \subset \operatorname{cl}_{V}(D \cap V)$. But then $d(V) \leqslant|D| \leqslant \alpha$, contrary to 1.5 above.

The proof is complete.

The following consequence of Theorem 2.1 was proved by R. Levy and R. H. McDowell [3] in the case $\omega \leqslant \gamma \leqslant 2^{\omega}$; they asked, in effect, if the result could be achieved for $2^{\omega}<\gamma \leqslant 2^{2^{\omega}}$. We note that in our abstract [2] we have outlined a proof of Corollary 2.2 based on the Levy-McDowell method of [3]; this method is quite different from those of the present paper.

2.2. Corollary. If $\omega \leqslant \gamma \leqslant 2^{2^{\omega}}$, there is a separable space equal to the (appropriately topologized) disjoint union of $\gamma$ copies of the space $U(\omega)$.

3. A final remark. It is tempting to believe that for every collection $\left\{X_{\xi}\right.$ : $\xi<\gamma\}$ of spaces such that $\gamma<\log 2^{\alpha}$ and $d\left(X_{\xi}\right)>\alpha$ for all $\xi<\gamma$, the disjoint union $X=\cup_{\xi<\gamma} X_{\xi}$ admits no topology such that $d(X) \leqslant \alpha$ and each $X_{\xi}$ is a topological subspace. The following simple example, though susceptible to substantial generalization, is sufficient to dispel this belief. Additional examples are expected in [4].

3.1. TheOREM. Let $\alpha$ and $\gamma$ be cardinals with $\alpha \geqslant \omega$ and with $2 \leqslant \gamma \leqslant 2^{2^{\alpha}}$, and for $\xi<\gamma$ let $X_{\xi}$ be a discrete space such that $\left|X_{\xi}\right|=\alpha^{+}$. Then the set-theoretic disjoint union $X=\cup_{\xi<\gamma} X_{\xi}$ admits a topology such that $d(X)=\alpha$ and each $X_{\xi}$ is a topological subspace of $X$.

Proof. Since $w\left(X_{\xi}\right)=\alpha^{+} \leqslant 2^{\alpha}$, the case $\log 2^{\alpha} \leqslant \gamma$ is handled by Theorem 2.1. We assume in what follows that $\gamma \leqslant \alpha$.

Let $f$ be a fixed-point-free permutation of $\gamma$, for $\xi<\gamma$ choose $D_{\xi} \subset X_{\xi}$ such that $\left|D_{\xi}\right|=\alpha$, and identify $X_{f(\xi)} \backslash D_{f(\xi)}$ with a (discrete) family of uniform ultrafilters over the discrete space $D_{\xi}$. (Such a family exists by 1.5 above.) Writing

$$
Y_{\xi}=D_{\xi} \cup\left(X_{f(\xi)} \backslash D_{f(\xi)}\right)
$$

we have the topological inclusion $D_{\xi} \subset Y_{\xi} \subset \boldsymbol{\beta}\left(D_{\xi}\right)$, so that $d\left(Y_{\xi}\right)=\alpha$. Now let $X$ be the topological disjoint union of the spaces $Y_{\xi}$-i.e., a subset $S$ of $X$ is open if and only if $S \cap Y_{\xi}$ is open in $Y_{\xi}$ for each $\xi<\gamma$. It is clear that $d(X)=\alpha$. Finally for $\xi<\gamma$ there is $\eta<\gamma$ such that $\eta \neq \xi$ and $\xi=f(\eta)$; 
since $D_{\xi}$ and $X_{\xi} \backslash D_{\xi}$ are disjoint discrete subsets of the disjoint open-andclosed subspaces $Y_{\xi}$ and $Y_{\eta}$ respectively, the set $X_{\xi}$ is discrete in $X$, as required.

\section{REFERENCES}

1. W. W. Comfort and S. Negrepontis, The theory of ultrafilters, Springer-Verlag, Berlin and New York, 1974.

2. W. W. Comfort and Teklehaimanot Retta, Separable unions of copies of $\beta N \backslash N$, Notices Amer. Math. Soc. 22 (1975), A-716. Abstract \# 75T-G121.

3. R. Levy and R. H. McDowell, Dense subsets of $\beta X$, Proc. Amer. Math. Soc. 50 (1975), 426-430. MR 51 \#6733.

4. Teklehaimanot Retta, Doctoral dissertation, Wesleyan Univ., 1977.

Department of Mathematics, Wesleyan University, Middletown, Connecticut 06457 